\title{
Does the Penrose Suggestion as to Black Holes from a Prior Universe Showing Up in Today's Universe Have Credibility? Examined from a Singular, and Nonsingular Beginning of Cosmological Expansion
}

\author{
Andrew Walcott Beckwith \\ Physics Department, Chongqing University, College of Physics, Chongqing University Huxi Campus, \\ Chongqing, China \\ Email: Rwill9955b@gmail.com, abeckwith@uh.edu
}

How to cite this paper: Beckwith, A.W. (2021) Does the Penrose Suggestion as to Black Holes from a Prior Universe Showing Up in Today's Universe Have Credibility? Examined from a Singular, and Nonsingular Beginning of Cosmological Expansion. Journal of High Energy Physics, Gravitation and Cosmology, 7, 149-171.

https://doi.org/10.4236/jhepgc.2021.71008

Received: October 22, 2020

Accepted: January 10, 2021

Published: January 13, 2021

Copyright $\odot 2021$ by author(s) and Scientific Research Publishing Inc. This work is licensed under the Creative Commons Attribution International License (CC BY 4.0).

http://creativecommons.org/licenses/by/4.0/ (c) (i) Open Access

\begin{abstract}
We examine if there are grounds to entertain the Penrose suggestion as to black holes from a prior cycle of creation appearing in the present cosmos. There are two cases to consider. One a singular start to the Universe or as Karen Freeze and others have modeled a non-singular start. The two cases are different and touch upon the limits of validity of the Penrose singularity theorem. We will first of all state the two cases, singular and nonsingular, and then afterwards, briefly allude to the Penrose singularity theorem. The plausibility of the singular cosmological expansion start point $\mathrm{w}$ case analysis of Black holes from a prior universe will be discussed first Afterwards, a synopsis of the Penrose singularity theorem. After that, the Nonsingular case of a starting point of the expansion of the Universe will be entertained and described. Since the nonsingular start to the expansion of the Universe is not so well known, a considerable amount of space will be spent upon what I view as mathematical constructions allowing for its analysis. About the only way to ascertain these cases will be by GW astronomy, hence the details of GW production from the early Universe will be covered in excruciating detail. The methodology for that section is simple. Use a construction for a minimal time-step, then from there get emergent space-time conditions for a bridge from a nonsingular start to the universe, to potential Quantum gravity conditions. Our Methodology is to construct using a "trivial" solution to massive gravitons, and a nonsingular start for expansion of the universe. Our methodology has many unintended consequences, not the least is a relationship between a small timestep, which is called $t$, and then the minimum scale fac-
\end{abstract}


tor and even the tension or property values of the initial space-time wall, all of which are a consequence of a "trivial" solution taking into account "massive" gravitons. From there we next will in future articles postulate conditions for experimental detectors for subsequent data sets to obtain falsifiable data sets. Finally upon doing this, the outlines of the way to ascertain data sets as to either falsify or confirm the Penrose suggestion will be the final concluding part of the manuscript.

\section{Keywords}

Minimum Scale Factor, Cosmological Constant, Space-Time Bubble, Penrose Singularity, Prior Universe Black Holes

\section{First Let Us View the Penrose Suggestion as to Black Holes from a Prior Universe}

In order to see this, consider a suggestion as to black holes, being the template for a start to the present universe given by [1], and also [2] which has the Penrose suggestion of an imprint of a prior Universe black hole having an effect upon the CMBR spectrum. The CMBR spectrum is a real datum, but the worth of getting this information would be in terms of having what was said in [2] as to the "ghost" of prior universe black hole radiation. This idea is presented in our Figure 1, which is copied from page 2 of reference [2].

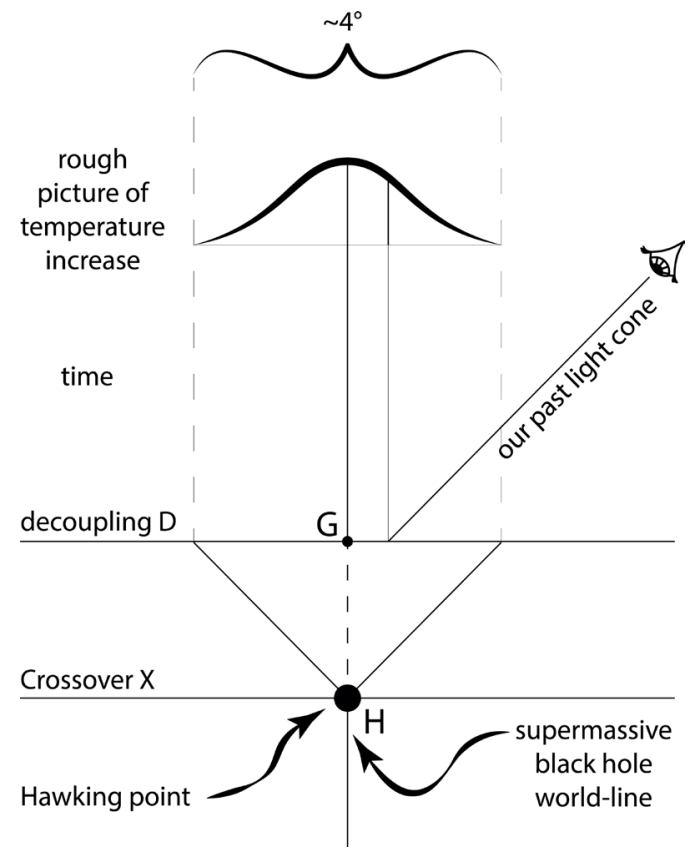

Figure 1. A conformal diagram representing the effect of a highly energetic event occurring at the space-time point $\mathrm{H}$. In CCC, $\mathrm{H}$ is taken to be a Hawking point, where virtually the entire Hawking radiation of a previous-aeon supermassive black hole is concentrated at $\mathrm{H}$ by the conformal compression of the hole's radiating future. 
i.e. the image of a supermassive black hole is then superimposed upon the $\mathrm{CMBR}$ and then the question is, does this have any other effects than a signature on the CMBR?

See a summary of this in [3] which is a way of saying that the CMBR can have traces, via CCC recycling of Black holes.

The CMBR can be affected by Gravitational radiation, as seen in [4], with more details in [5]. We argue in doing all this that we have in actuality two choices, the first one, on if we have a singular start to the universe, as given by the Penrose singularity theorem as given in [6], and the next one if we have a nonsingular modification of the Friedman equation as given in [7].

Having a non singular scale factor will allow for the $2^{\text {nd }}$ case which we argue is in tandem with the treatment of the ghosts of the supermassive black holes is a way to initiate putting the ghosts of prior supermassive black holes into the present CMBR, which has to be reconciled with the generalized facts given by Zeldovich, in [8], and congruent with [9] [10].

\section{The Basic Facts of the Penrose Singularity Theorem and Why We Do Not Assume a Point Scale Factor, as an Initial Starting Point}

\section{[6] has this foundational structure which is relevant to out inquiry Quote}

8.2. Quantum effects a very important line of research arises from the tension between the singularity theorems and the (yet unfound) theory of quantum gravity. It is widely accepted that the existence of classical singularities signal a breakdown of the classical theory at extreme conditions, which is precisely when gravitational quantum effects will become relevant. Thus, there is a need to clarify if the singularity theorems, or part of them, can survive when entering into a quantum regime, or if they then simply vanish altogether. For a general discussion, see [35]. A first step towards the analysis of singularity theorems in this respect is the weakening of the "energy conditions"-also relevant in the classical Singularity theorems 38 regime, that is to say, finding an appropriate version of the curvature condition in the theorems. Early results in this direction include the theorems based on averaged energy conditions [303] as discussed in subsection 5.1, which were used to deal with the quantum violations of the energy conditions in [264], later improved in [265]. A larger discussion can be found in [110] (and references therein) and has been recently newly considered in [109], where an analysis of Raychadhuri-like equations is performed proving that it is viable to have energy-momentum tensors which fail to satisfy even averaged energy conditions as long as an appropriate version of them with an exponential damping factor are in place. This leads to a proof of a version of the Penrose singularity theorem allowing for global violations of the energy conditions. 
End of quote

What we will be analyzing is a definite quantum violation of the energy condition as given by [11].

Having said that, [12] has some of the basic facts to consider with a statement of the Euclidian Universe which is very pertinent to our situation.

\section{Quote}

Unlike the black hole pair creation, one couldn't say that the de Sitter universe was created out of field energy in a pre-existing space. Instead, it would quite literally be created out of nothing: not just out of the vacuum but out of absolutely nothing at all because there is nothing outside the universe. In the Euclidean regime, the de Sitter universe is just a closed space like the surface of the Earth but with two more dimensions. If the cosmological constant is small compared to the Planck value, the curvature of the Euclidean four sphere should be small. This will mean that the saddle point approximation to the path integral should be good, and that the calculation of the wave function of the universe won't be affected by our ignorance of what happens in very high curvatures.

End of quote

Our point of divergence from this statement is

A. We do NOT assume that the Universe is made out of "nothing" because we assume via a version of CCC cosmology [13] that there has been regular inputs into this present universe;

B. We do assume that we may have a wavefunction of the Universe which does not take into accord high curvature regimes;

C. We also though in making the wavefunction of the Universe assume what was given in [7] so as to avoid having a scale factor involving a singular point, which would make our suppositions unworkable.

\section{Stating the Wave Function Treatment of Acceleration from the Initial Phase Transition}

In a word we are assuming that there is a marked phase transition between early time pre expansion physics, and then the expansion physics. To do so we are looking at [14]. i.e. what we are doing is akin to the following description which was pitched to be relevant to the electroweak regime. With certain caveats, we hold that it is similar to what we would have at the very start of expansion of the universe. i.e. change the description from the electroweak to the initial nonsingular starting point of the universe in line with [7] and we are set to go for our cosmological evolution. See [15].

Quote

Phase transitions are a generic, but not universal, feature of gauge field theories, like the Standard Model, which are based on elementary particle 
mass generation by spontaneous symmetry-breaking [1] [2]. When there is a phase transition in a gauge theory it is (except for special parameter choices) first-order, which means that just below the critical temperature, the universe transitions from a metastable quasi-equilibrium state into a stable equilibrium state, through a process of bubble nucleation, growth, and merger [3] [4] [5] [6]. Such a first-order phase transition in the early universe naturally leads to the production of gravitational waves [7] [8]. If it took place around the electroweak scale, by which we mean temperatures in the range $100-1000 \mathrm{GeV}$, the gravitational wave signal could lie in the frequency range of the upcoming space-based gravitational wave detector LISA (Laser Interferometer Space Antenna) [9]. The approval of the mission, and the detection of gravitational waves [10], has generated enormous interest in phase transitions in the early universe.

End of quote

The evolution of energy section, in [14], about pp. 34-39 will be obeyed once we commence once we get through the initial phase transitions. In the mean time, once we get to the initial phase transitions, [15] we have that [16] describes well the initial gravitational wave generation well.

We are concerned though about if there is not just CMBR support for the Penrose conjecture [1] [2] [3] and [4] but also GW signatures from Pre Big Bang black holes disturbing the COMBR leading to gravitational signatures. which outline this addendum to classical GR.

[17] Hee-Boon Low and Chi Xiong, outline on page 60, Equation (13) an additional term, Vinens equation for a vortex tangle to be added to the usual cosmological evolution equations, which may account for what was brought up in [18], namely a reconciliation of the Higgs hypothesis given in pp35 to 44, but that would be a bridge for the creation of matter-energy after a first order phase transition. What we will be concerned with next will be the bridge from CCP inputs into the generation of the present cosmos, to our present universe. From between a CCC cosmology construction, with an additional modification put in, to the physics of the question we put in initially which will be of with regards to signatures of Black Holes, from a prior universe to our present cosmos. This will be the reason to use [7] [19] [20].

See [1] [2] [3] as to what we are trying to ascertain exists in terms of GW signatures for black holes in the CMBR [7] [20] whereas referencing the superfluid universe, as a candidate, after the phase transition [19] The phase transition details, plus preserving the inputs from [7] will be the conclusion of this manuscript.

\section{Emergent Tunneling Wave Space-Time Equation Used to Compare the Start of Expansion}

We use the construction from [21] as to, if the initial "potential" $V(\phi)$ is very 
large, how to isolate the form of the wavefunction, especially if $a^{2} V(\phi)>1$, even if $a$ is the initial value, i.e. very, very small, even if $a_{\min } \propto 5.13 \times 10^{-62}$, and then by page 269 of [21] go to the following formulation. Namely that we look at

$$
\Psi_{T} \propto \frac{\exp (-1 / 3 V(\phi))}{\left(a^{2} V(\phi)-1\right)^{1 / 4}} \cdot \exp \left(-i \cdot \frac{\left(a^{2} V(\phi)-1\right)^{3 / 2}}{3 V(\phi)}\right)
$$

Our entries into all the above will be the subject of the next several sessions of our document and we will endeavor to explain how this all fits into the early universe modeling we will be working with as far as foundational research into Quantum gravity.

In terms of a contribution from the modified Einstein Equations, we will be considering the time components of the Einstein equation with the stress and strain component in quantum expectation language, i.e. as in Colins, Martin and Squires, written as, due to emergent space-time from pre Planckian to Planckian regimes. i.e. look at the time related transitions, and this is what we get. i.e. the basic equations are related as follow [22]

$$
\begin{aligned}
& \left(\frac{R_{\mu \nu}-\frac{R}{2} \cdot g_{\mu \nu}+\Lambda g_{\mu \nu}}{8 \pi G_{N}}+\left\langle\Psi\left|T_{\mu \nu}\right| \Psi\right\rangle=0\right) \\
& \underset{\mu, v \rightarrow 0,0}{\longrightarrow} \frac{R_{00}-\frac{R}{2} \cdot g_{00}+\Lambda g_{00}}{8 \pi G_{N}}+\left\langle\Psi\left|T_{00}\right| \Psi\right\rangle=0
\end{aligned}
$$

Here we will have the following approximations, used. Namely look at

$$
\rho \approx \frac{\dot{\phi}^{2}}{2}+V(\phi) \equiv \frac{\gamma}{8 \pi G} \cdot t^{2}+V_{0} \cdot\left\{\sqrt{\frac{8 \pi G V_{0}}{\gamma(3 \gamma-1)}} \cdot t\right\}^{\sqrt{\frac{\gamma}{4 \pi G}}-\sqrt{\frac{8 \pi G}{\gamma}}}
$$

which are a result of the following equations used when we are looking at a tunneling Wave Space-time equation. Then, using this procedure above is using Padmabhan's inputs into the inflaton potential and also getting initial spacetime data into the inflaton itself, as given in [23].

Which uses at the surface of a presumed non singular start to the expansion of the universe.

$$
a(t)=a_{\min } t^{\gamma}
$$

Leading to [23] the inflaton [24].

$$
\phi \approx \sqrt{\frac{\gamma}{4 \pi G}} \ln \left[\sqrt{\frac{8 \pi V_{0} G}{\gamma \cdot(3 \gamma-1)}} \cdot t\right]
$$

And what we will use later the "inflaton potential" we write as [25].

$$
V=V_{0} \cdot\left\{\sqrt{\frac{8 \pi G V_{0}}{\gamma(3 \gamma-1)}} \cdot t\right\}^{\sqrt{\frac{\gamma}{4 \pi G}}-\sqrt{\frac{8 \pi G}{\gamma}}}
$$

Furthermore, we have from [24] pp. 212-213, and [25]. 


$$
m \cdot \partial_{t}\left(a^{2}-a^{3}\right)=0
$$

And then, a minimum time step we define via a minimum time step of

$$
t=\left(\frac{2}{3 a_{\min }}\right)^{1 / \gamma}
$$

Note that if the time as defined by Equation (8) is on the order of Planck time, i.e. $10^{-44}$ seconds, we have then that $\gamma \approx 61-62$.

We then close with a statement, if

$$
\begin{aligned}
& \frac{3\left(\left(\frac{\ddot{a}}{a}\right) \cdot\left(g_{00}-1\right)+\left(\frac{\dot{a}}{a}\right)^{2} g_{00}+\frac{\kappa}{a^{2}} g_{00}\right)+\Lambda g_{00}}{8 \pi G_{N}} \\
& =-\left\langle\Psi\left|\frac{c \dot{\phi}^{2}}{2}+c V(\phi) \equiv \frac{c \gamma}{8 \pi G} \cdot t^{2}+c V_{0} \cdot\left\{\sqrt{\frac{8 \pi G V_{0}}{\gamma(3 \gamma-1)}} \cdot t\right\}^{\sqrt{\frac{\gamma}{4 \pi G}}-\sqrt{\frac{8 \pi G}{\gamma}}|\Psi\rangle}\right| \Psi\right\rangle
\end{aligned}
$$

If $t$ he wave function is taken via Equation (1) put in, we have that the effective wave function for the "Quantum" part in the RHS of Equation (9) is then

$$
\Psi_{T} \propto \frac{\exp (-1 / 3 V(\phi))}{\left(a^{2} V(\phi)-1\right)^{1 / 4}}
$$

Whereas we will be looking at the following approximation for the right hand side of Equation (10), namely

$$
\begin{aligned}
& \frac{R_{00}-\frac{R}{2} \cdot g_{00}+\Lambda g_{00}}{8 \pi G_{N}}+\left\langle\Psi\left|T_{00}\right| \Psi\right\rangle \\
& =\frac{3\left(\left(\frac{\ddot{a}}{a}\right)\left(g_{00}-1\right)+\left(\frac{\dot{a}}{a}\right)^{2} g_{00}+\frac{\kappa}{a^{2}} g_{00}\right)+\Lambda g_{00}}{8 \pi G_{N}} \\
& +\left\langle\Psi\left|\frac{c \gamma}{8 \pi G} \cdot t^{2}+c V_{0} \cdot\left\{\sqrt{\frac{8 \pi G V_{0}}{\gamma(3 \gamma-1)}} \cdot t\right\}^{\sqrt{\frac{\gamma}{4 \pi G}}}-\sqrt{\frac{8 \pi G}{\gamma}}\right| \Psi\right\rangle
\end{aligned}
$$

The above Equation (11) is set equal to zero.

Hence also note we are going to do the following for breaking down the parts of what would otherwise be an ENORMOUS integrand expression to evaluate. Namely the midpoint rule for numerical evaluation of the integral, using [26]. We will justify this procedure due to the "radius" of the initial "space-bubble" is so close to a singularity, and we are talking about emergent spacetime. Hence, this idea as of [26] is used whereas I would NEVER otherwise even think of it. The centering of the initial problem, about its time component should also be seen in the context that the idea as will be used in further developments is that time, within the space-time bubble itself does NOT exist, and that there is instead a construction of time on the SURFACE of the initial near singularity.

The author is well aware of the Penrose singularity theorem [27]. At the end 
of this document, there will be a discussion of where I think it (the singularity theorem) holds, and what is missed, which is part of the multiverse discussion which is explicitly referred to and put in our text, in order to achieve having a constant value of $\hbar$ cosmological cycle per cycle without referencing the insanity of the Anthropic principle which has been used by Tippler and others to insert a religious dimension into Cosmology, which I view as missing the point entirely as to the evolution of the Universe itself [28]. This is different from an argument given by Tegmark, as of [29] which gives a surprisingly well motivated argument as to spatial dimensions. I think the argument is well worth investigating. But the Strong Antropic principle is quite another matter entirely, to be avoided.

$$
\begin{aligned}
& \left\langle\Psi\left|\frac{c \gamma}{8 \pi G} \cdot t^{2}+c V_{0} \cdot\left\{\sqrt{\frac{8 \pi G V_{0}}{\gamma(3 \gamma-1)}} \cdot t\right\}^{\sqrt{\frac{\gamma}{4 \pi G}}}-\sqrt{\frac{8 \pi G}{\gamma}}\right| \Psi\right\rangle=A_{1}+A_{2} \\
& A_{1}=\left\langle\Psi\left|\frac{c \gamma}{8 \pi G} \cdot t^{2}\right| \Psi\right\rangle \approx \ell_{\text {Planck }}^{3} \int \mathrm{d} t\left\{\frac{\exp (-2 / 3 V(\phi))}{\left(a^{2} V(\phi)-1\right)^{1 / 2}} \cdot \frac{c \gamma}{8 \pi G} \cdot t^{2}\right\} \\
& A_{2}=\ell_{\text {Planck }}^{3} \int \mathrm{d} t\left\{\frac{\exp (-2 / 3 V(\phi))}{\left(a^{2} V(\phi)-1\right)^{1 / 2}} \cdot c V_{0} \cdot\left\{\sqrt{\frac{8 \pi G V_{0}}{\gamma(3 \gamma-1)}} \cdot t\right\}^{\sqrt{\frac{\gamma}{4 \pi G}}} \cdot \sqrt{\frac{8 \pi G}{\gamma}}\right. \\
& a=a_{\text {min }} t^{\gamma} \\
& \left.V=V_{0} \cdot\left\{\sqrt{\frac{8 \pi G V_{0}}{\gamma(3 \gamma-1)}} \cdot t\right\}^{\sqrt{\frac{\gamma}{4 \pi G}}-\sqrt{\frac{8 \pi G}{\gamma}}}\right\}
\end{aligned}
$$

From there we will use simple approximations to ascertain as to how to analyze Equation (11) and Equation (12) and proceed with the rest of this article mainly using the midpoint rule for numerical approximation.

To do so, we look at the following approximation, namely if

$$
\left.\begin{array}{c}
A_{1}=\left\langle\Psi\left|\frac{c \gamma}{8 \pi G} \cdot t^{2}\right| \Psi\right\rangle \approx \ell_{\text {Planck }}^{3} \int \mathrm{d} t\left\{\frac{\exp (-2 / 3 V(\phi))}{\left(a^{2} V(\phi)-1\right)^{1 / 2}} \cdot \frac{c \gamma}{8 \pi G} \cdot t^{2}\right\} \\
\approx \ell_{\text {Planck }}^{3} t_{\text {Planck }}\left\{\frac{\exp \left(-2 / 3 V\left(\frac{t_{\text {Planck }}}{2}\right)\right)}{\left(a^{2} V\left(\frac{t_{\text {Planck }}}{2}\right)-1\right)^{1 / 2}} \cdot \frac{c \gamma}{8 \pi G} \cdot \frac{t_{\text {Planck }}^{2}}{4}\right\} \\
A_{2}=\ell_{\text {Planck }}^{3} \int \mathrm{d} t\left\{\frac{\exp (-2 / 3 V(\phi))}{\left(a^{2} V(\phi)-1\right)^{1 / 2}} \cdot c V_{0} \cdot\left\{\sqrt{\frac{8 \pi G V_{0}}{\gamma(3 \gamma-1)}} \cdot t\right\}^{\sqrt{\frac{\gamma}{4 \pi G}}}-\sqrt{\frac{8 \pi G}{\gamma}}\right\} \\
\approx \ell_{\text {Planck }}^{3} t_{\text {Planck }}\left\{\frac{\exp \left(-2 / 3 V\left(\frac{t_{\text {Planck }}}{2}\right)\right)}{\left(a^{2} V\left(\frac{t_{\text {Planck }}}{2}\right)-1\right)^{1 / 2}} \cdot c V_{0} \cdot\left\{\sqrt{\frac{8 \pi G V_{0}}{\gamma(3 \gamma-1)}} \cdot \frac{t_{\text {Planck }}}{2}\right\}\right.
\end{array}\right\}
$$


Also

$$
V\left(\frac{t_{\text {Planck }}}{2}\right)=V_{0} \cdot\left\{\sqrt{\frac{8 \pi G V_{0}}{\gamma(3 \gamma-1)}} \cdot \frac{t_{\text {Planck }}}{2}\right\}^{\sqrt{\frac{\gamma}{4 \pi G}}-\sqrt{\frac{8 \pi G}{\gamma}}}
$$

Also

$$
a^{2} V\left(\frac{t_{\text {Planck }}}{2}\right)=a_{\text {min }}^{2} t^{2 \gamma} \cdot V_{0} \cdot\left\{\sqrt{\frac{8 \pi G V_{0}}{\gamma(3 \gamma-1)}} \cdot \frac{t_{\text {Planck }}}{2}\right\}^{\sqrt{\frac{\gamma}{4 \pi G}}-\sqrt{\frac{8 \pi G}{\gamma}}}
$$

In addition the fact I will refer to a very strong initial domain wall, of a boundary between the early universe, and also state the following.

Pick the following in order to insure the initial wave function as given by Equation (1) remains valid within the context of a bubble of space-time at the start of inflation, namely have:

$$
\begin{aligned}
& a^{2} V\left(\frac{t_{\text {Planck }}}{2}\right)=a_{\text {min }}^{2} t_{\text {Planck }}^{122} \cdot V_{0} \cdot\left\{\sqrt{\frac{8 \pi G V_{0}}{61 \cdot(183-1)}} \cdot \frac{t_{\text {Planck }}}{2}\right\}^{\sqrt{\frac{61}{4 \pi G}}-\sqrt{\frac{8 \pi G}{61}}}>1 \\
& \Rightarrow V_{0}^{1+\sqrt{\frac{8 \pi G}{61}}-\sqrt{\frac{61}{4 \pi G}}}>\frac{1}{\left\{a_{\text {min }}^{2} t_{\text {Planck }}^{122} \cdot\left\{\sqrt{\frac{8 \pi G}{61 \cdot(183-1)}} \cdot \frac{t_{\text {Planck }}}{2}\right\}^{\sqrt{\frac{61}{4 \pi G}}-\sqrt{\frac{8 \pi G}{61}}}\right\}}
\end{aligned}
$$

This restriction is in tandem with having $\mathrm{t}$ to the $61^{\mathrm{st}}$ power at the start of expansion of the Planckian space-time regime as reflecting the hyper rapid onset of inflation itself.

\section{Utilizing Equation (2) and Equation (3), as to Nonsingular Friedman Equations to Obtain One Value for the Cosmological Constant}

To do this, we will bring up several considerations about the Cosmological Constant, within our framework of a non singular start to the expansion of our universe.

A given in our work is that within the nucleation of space-time, that time, even as given in Equation (2) simply does not exist, but that we will be able to use the results of Freeze [30] as far as a nonsingular Friedman equation to come up with

$$
H=\frac{\dot{a}}{a}=\sqrt{\frac{8 \pi}{3 M_{P}^{2}}} \cdot \sqrt{\left(\rho-\frac{\rho^{2}}{2|\sigma|}\right)}
$$

The easiest thing to do would be to make the following identification, namely writing a quadratic equation in terms of density, which we would write as the following form, namely for an unspecified for now $g_{00}$ the following quadratic equation in terms of a general density function which we would write as 


$$
\begin{aligned}
& 3\left(\left(\gamma \cdot(\gamma-1) t^{\gamma-1}\right) \cdot\left(g_{00}-1\right)+\frac{8 \pi}{3 M_{P}^{2}}\left(\rho-\frac{\rho^{2}}{2|\sigma|}\right) g_{00}+\frac{\kappa}{a^{2}} g_{00}\right)+\Lambda g_{00} \\
& =-\left\langle\Psi\left|\frac{c \gamma}{8 \pi G} \cdot t^{2}+c V_{0} \cdot\left\{\sqrt{\frac{8 \pi G V_{0}}{\gamma(3 \gamma-1)}} \cdot t\right\}^{\sqrt{\frac{\gamma}{4 \pi G}}-\sqrt{\frac{8 \pi G}{\gamma}}}\right| \Psi\right\rangle
\end{aligned}
$$

Then we could write the following, namely

$$
\begin{aligned}
& \left(\rho-\frac{\rho^{2}}{2|\sigma|}\right)+\frac{\Lambda \cdot M_{P}^{2}}{8 \pi}+\frac{\kappa \cdot M_{P}^{2}}{a^{2} \cdot 8 \pi}+\left(\gamma \cdot(\gamma-1) t^{\gamma-1}\right) \cdot \frac{\left(g_{00}-1\right) \cdot M_{P}^{2}}{g_{00} \cdot 8 \pi} \\
& +\left\langle\Psi\left|\frac{c \gamma}{8 \pi G} \cdot t^{2}+c V_{0} \cdot\left\{\sqrt{\frac{8 \pi G V_{0}}{\gamma(3 \gamma-1)}} \cdot t\right\} \sqrt{\frac{\gamma}{4 \pi G}}-\sqrt{\frac{8 \pi G}{\gamma}}\right| \Psi\right\rangle \frac{M_{P}^{2}}{g_{00} \cdot 8 \pi}=0
\end{aligned}
$$

We will from here isolate the Cosmological constant, which we write as

$$
\begin{aligned}
\Lambda= & \frac{8 \pi}{M_{P}^{2}}\left(\frac{\rho^{2}}{2|\sigma|}-\rho\right)+\frac{\kappa \cdot M_{P}^{2}}{a^{2} \cdot 8 \pi}+\left(\gamma \cdot(\gamma-1) t^{\gamma-1}\right) \cdot \frac{\left(1-g_{00}\right)}{g_{00}} \\
& -\frac{1}{g_{00}}\left\langle\Psi\left|\frac{c \gamma}{8 \pi G} \cdot t^{2}+c V_{0} \cdot\left\{\sqrt{\frac{8 \pi G V_{0}}{\gamma(3 \gamma-1)}} \cdot t\right\}^{\sqrt{\frac{\gamma}{4 \pi G}}-\sqrt{\frac{8 \pi G}{\gamma}}}\right| \Psi\right\rangle
\end{aligned}
$$

The simplification if we have, say $g_{00} \rightarrow 1$ with $\kappa$ nonzero come up with

$$
\begin{aligned}
\Lambda \approx & \frac{8 \pi}{M_{P}^{2}}\left(\frac{\rho^{2}}{2|\sigma|}-\rho\right)+\frac{\kappa \cdot M_{P}^{2}}{a^{2} \cdot 8 \pi} \\
& -\left\langle\Psi\left|\frac{c \gamma}{8 \pi G} \cdot t^{2}+c V_{0} \cdot\left\{\sqrt{\frac{8 \pi G V_{0}}{\gamma(3 \gamma-1)}} \cdot t\right\} \sqrt{\frac{\gamma}{4 \pi G}}-\sqrt{\frac{8 \pi G}{\gamma}}\right| \Psi\right\rangle
\end{aligned}
$$

We will, instead, in comparing this value of the cosmological constant go to a method of comparing two first integrals, and from there compare that value with Equation (21) and Equation (22).

\section{Why Is a Nonsingular Start to Cosmology Important}

In Figure 2 we copy what was done by Ashtekar, in Zelsovich 4 as to what was part of anisotropic fits to the $\mathrm{E}$ and $\mathrm{B}$ polarization, as given.

Questioning by me, the attendee of the Zeldovich 4 conference (I had my own presentation) revealed that the correction Abhay inserted to the left of the "temperatire" spectrum was not decisively impacted in his LQG treatment of the E and B polarizations. I view the item as far from closed, and I also told Zeldovich 4 that a non singular start to the expansion of the universe makes this problem far more tractable. FTR.

We review [31] and in and in addition look at [32] I also proceed to Klauder Enhanced quantization and the Cosmological constant which is in the next section. 


\section{Klauder Enhanced Quantization and the Cosmological Constant}

Our methodology is to compare what we are doing with the work done by the author initially without enhanced quantization and to argue that the results are qualitatively a close match, with the Klauder.

We are going to go to page 78 by Klauder [33] [34] of what he calls on page 78 a restricted Quantum action principle which he writes as: $S_{2}$ where we write a 1-1 equivalence as in [23] [32] [33] [34].

$$
\begin{aligned}
& S_{2}=\int_{0}^{T} \mathrm{~d} t \cdot\left[p(t) \dot{q}(t)-H_{N}(p(t), q(t))\right] \\
& \approx S_{1}=\frac{1}{2 \kappa} \cdot \int \sqrt{-g} \cdot \mathrm{d}^{4} x \cdot(\Re-2 \Lambda)
\end{aligned}
$$

Our assumption is that $\Lambda$ is a constant, hence we assume then the following approximation, from [12] [32].

$$
\begin{aligned}
& \frac{p_{0}^{2}}{2}=\frac{p_{0}^{2}(N)}{2}+N \text {; for } 0<N \leq \infty \text { and } q=q_{0} \pm p_{0} t \\
& V_{N}(x)=0 \text {; for } 0<x<1 \\
& V_{N}(x)=N \text {; otherwise } \\
& H_{N}(p(t), q(t))=\frac{p_{0}^{2}}{2}+\frac{(\hbar \cdot \pi)^{2}}{2}+N \text {; for } 0<N \leq \infty
\end{aligned}
$$

Our innovation is to then equate $q=q_{0} \pm p_{0} t \sim \phi$ and to assume small time step values. Then [32].

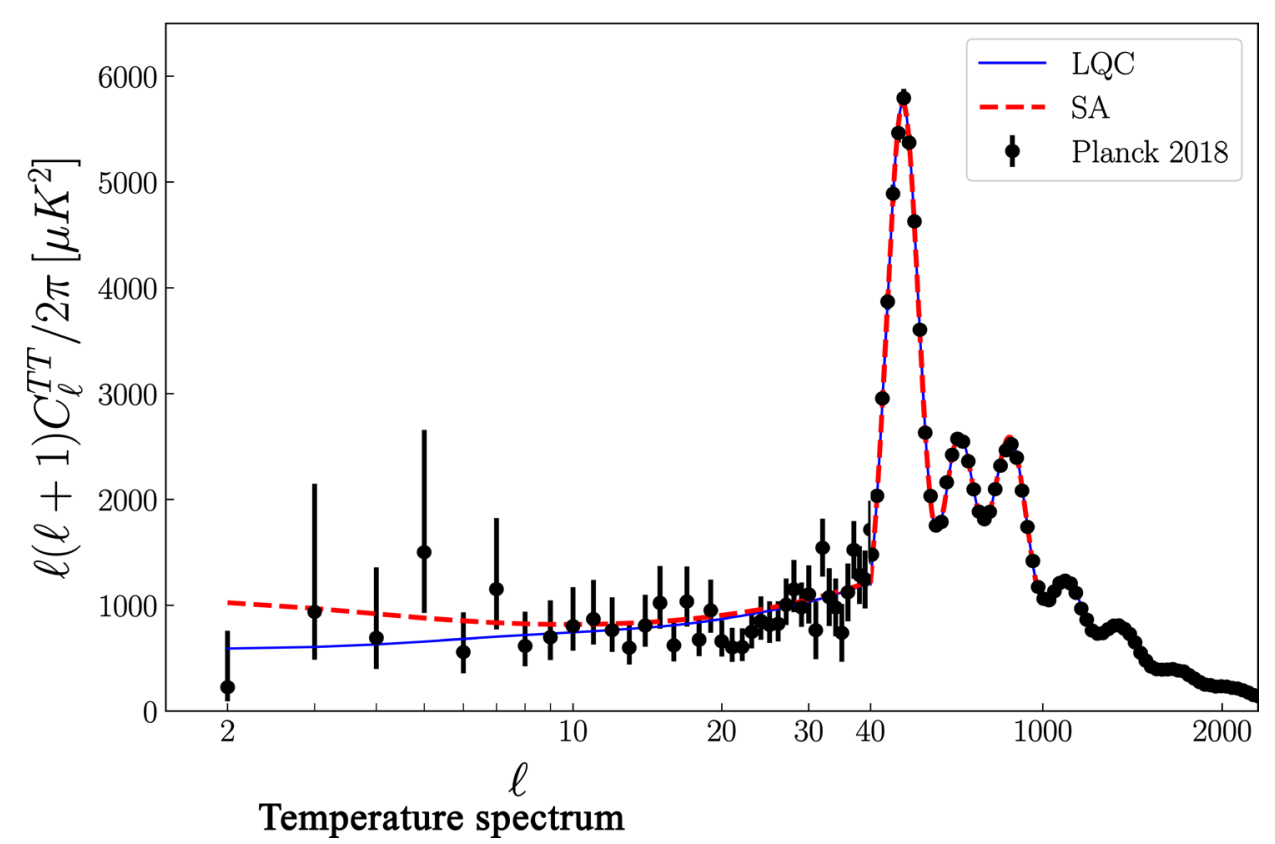

Figure 2. Now looking at what was discussed by Abhay Ashtekar in Zeldovich 4. On September 7, $2020[31]$. 


$$
\Lambda \approx \frac{-\left[\frac{V_{0}}{3 \gamma-1}+2 N+\frac{\gamma \cdot(3 \gamma-1)}{8 \pi G \cdot \tilde{t}^{2}}\right]}{\frac{1}{\kappa} \int \sqrt{-g} \cdot \mathrm{d}^{3} x}+\left.\left(6 \cdot\left(\frac{\ddot{a}}{a}+\left(\frac{\dot{a}}{a}\right)^{2}\right)\right)\right|_{t=\tilde{t}}
$$

Let us compare for a moment Equation (25) with what is derived in Equation (22). Depending upon what we put in for the density function $\rho$, what jumps out is that we have, then almost the same positive and negative contributions which will in fact lead to a nonperturbative solution to the Cosmological constant which we claim should, with further research converge to the same values. We will attend to this in the future. We will comment on comparing Equation (22) and Equation (25) in the end of the document with recommendations. See also [33] [34] and [35].

Now we have a candidate balance of contributions to $\Lambda$ at the start of the universe. Assuming $\mathrm{DE}$ is $\Lambda$ how do we account for a possible reduction of the role of DM? From the early universe?

According to Freese, Rindler-Daller Spolyar and Valluri massive BH of a mass $10^{8}$ solar masses formed early on, in the center of spiral galaxies, forming from DM stars as presented, here [35]. The point is this, that the following pie chart probably had more DM than today, with the Dark Stars perhaps shrinking the amount of DM and boosting the total budget of DR in the cosmos.

We do not exactly know what the DE is, but here is a clue as to its role in the present universe. And DM may have been considerably more prevalent than today, if the SM black holes from the center of galaxies are from giant DM stars then if Equation (22) and Equation (25) are prevalent DE, as we suggest,. Then the giant DM stars as postulated in [35]; [35] may be our preferred venue with the formation of elliptical galaxies in setting up conditions for reacceleration of the Universe. This is something to consider and is a research direction which the author will pursue in the future.

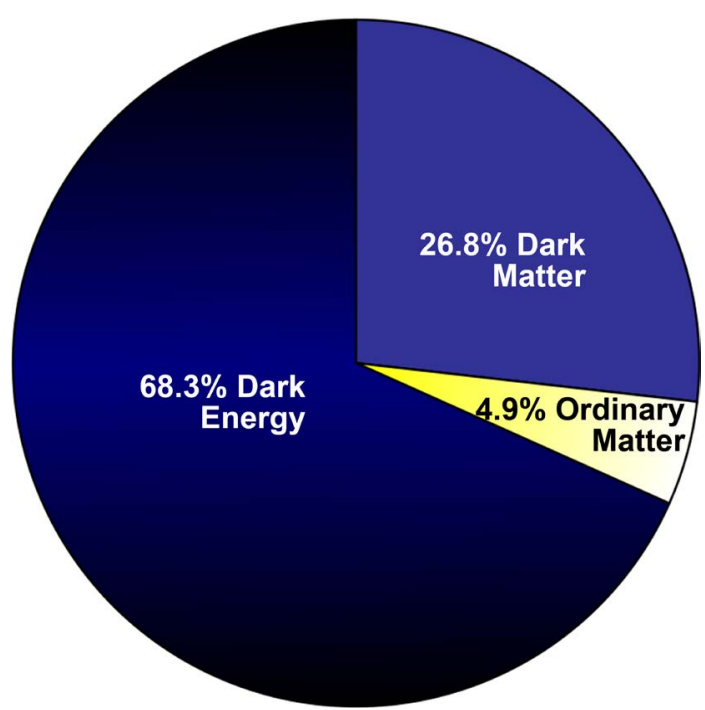

Figure 3. Attributed to commonly available descriptions [36] [37] [38] [39]. 


\section{Resetting Dark Energy as the Cosmological Constant and Reacceleration of the Universe 1 Billion Years Ago}

Here we are using inputs from [35] [36] [37] [38] [39], with [39] in particular] in particular by Peebles and Ratra giving the case that if the mathematics of [37] and [38] are taken care of, that indeed the Equation (22) and Equation (25) may be tending toward reacceleration of the universe, in line with a discussion given by Thanu Padmabhan in [23] which is simple and elegant. For additional work, we need to refer to [40] and the idea of a "jerk" calculation [41] [42] where re-acceleration of the universe is in tandem with the Jerk becoming negative valued, signifying re acceleration.

The author in [42] specifically did calculations as to the Jerk (re-acceleration) lead to a speed up of cosmological expansion, attributing this development to DE. What the author sees as a necessary extension of his research is to cile [36]-[41]; with the given phenomenology of [42] where reacceleration of the universe is stated to be a fact which should be investigated in terms of the cosmological constant, that is, if Equation (22) and Equation (25) are giving the same information. That has to be confirmed via rigorous calculations. Also, look at [43], and [44].

\section{Dark Energy Density as Affected by Equation (22) and Equation (25)}

We have also the discussion given in [35] and set $\kappa$ as spacetime curvature, and so then we have

$$
\rho_{D E}=\frac{3 \tilde{\alpha}}{8 \pi} \cdot\left(\dot{H}+2 H^{2}+\frac{\kappa}{a^{2}}\right)=-\frac{\tilde{\alpha}}{8 \pi} \cdot \mathfrak{R}
$$

In terms of the bubble of spacetime before inflation, we submit that time does not really exist and that then we will be considering a rewrite of the above as having, effectively $\dot{H}=0$. And the term $\rho_{D E}=\rho$. And if we apply Equation (26) we have within the bubble of spacetime This value for $\rho_{D E}=\rho$ at just about the surface of a bubble.

$$
\rho^{2}-\rho \cdot(2|\sigma|)\left(1-\left(\frac{3 \tilde{\alpha}}{8 \pi}\right)^{-1} \cdot\left(\frac{16 \pi}{3 M_{P}^{2}}\right)^{-1}\right)-\left(\frac{16 \pi}{3 M_{P}^{2}}\right)^{-1} \cdot(2|\sigma|) \cdot \frac{\kappa}{a^{2}}=0
$$

We can then use a minimum timestep defined by using [43] $\Delta E \Delta t \equiv \hbar$. If so then, if the initial volume is of the cube of a Planck length, we have a time step defined via, if the $\tilde{\alpha}$ value, were small, we would be probably be looking at

$$
\Delta t=\frac{l_{\text {Planck }}^{3} \hbar /|\sigma|}{\left(1-\left(\frac{3 \tilde{\alpha}}{8 \pi}\right)^{-1} \cdot\left(\frac{16 \pi}{3 M_{P}^{2}}\right)^{-1}\right) \pm \sqrt{\left(1-\left(\frac{3 \tilde{\alpha}}{8 \pi}\right)^{-1} \cdot\left(\frac{16 \pi}{3 M_{P}^{2}}\right)^{-1}\right)^{2}+\left(\frac{16 \pi}{3 M_{P}^{2}}\right)^{-1} \cdot(2|\sigma|)^{-1} \cdot \frac{8 \kappa}{a^{2}}}}
$$

A limiting solution, of all things turns out to be if the curvature term is small, 
with, among other approximations, to be, surprise, to have the bubble tension term dropping out. But this is not a GENERAL solution.

$$
\Delta t \approx 2 l_{\text {Planck }}^{3} \hbar \cdot \frac{\left(\left(\frac{3 \tilde{\alpha}}{8 \pi}\right)^{-1}-\left(\frac{16 \pi}{3 M_{P}^{2}}\right)\right)}{\frac{8 \kappa}{a^{2}}}
$$

\section{Does a Physics Equivalence between Equation (28) and Equation (29) Tie in with the Physics Stated in Equation $(8) ?$}

This really has to be ascertained. Equation (8) result is a very clean derivation involving a so called "trivial" solution to the massive gravity problem, which leads to yet another question to be confirmed, if possibly in experimental data sets. Does the following hold? In terms of massive Gravity? It is from [44] [24], Novello.

$$
m_{g}=\frac{\hbar}{c} \cdot \sqrt{\Lambda}
$$

The above formula (30) has to be confirmed and in some sense proved, as being not in conflict with Equation (22) and Equation (25) of this document.

\section{Does the Following Arguments Give Equivalent Information, to What We Obtain Comparing Equation} (22) and Equation (25)?

$$
\begin{gathered}
c \rho_{d S}=\left.\frac{\left(R_{00}-g_{00} R\right)}{8 \pi}\right|_{d S} \\
\left.\left.\Leftrightarrow \frac{\left(-3 \frac{\ddot{a}}{a}-g_{00} 6 \Lambda\right)}{8 \pi}\right|_{d S} \approx \frac{\left(-3 \frac{\ddot{a}}{a}+g_{00} 6\left(\frac{\ddot{a}}{a}+\left(\frac{\dot{a}}{a}\right)^{2}+\frac{2 \kappa}{a^{2}}\right)\right)}{8 \pi}\right|_{d S}
\end{gathered}
$$

See this final take away as to what the cosmological constant is equivalent to, i.e. does the following make sense? If we multiply Equation (31) say by the cube of a Planck volume, and then can we work with the following derivation?

$$
\Delta E \Delta t \approx \hbar \equiv \hbar \omega \Delta t \approx \hbar \omega \cdot\left(\frac{2}{3 a_{\min }}\right)^{1 / \gamma} \Rightarrow \omega \approx \hbar^{-1} \cdot\left(\frac{2}{3 a_{\min }}\right)^{-1 / \gamma}
$$

We think that this means that at the boundary of a space time bubble, that this would force us to have an enormous value for $\gamma$. We would also, if the initial energy were just at the boundary of the bubble of space time, have the odd situation for which we would have the following, namely at the surface of the bubble, so if one used Planck units with $l_{\text {Planck }} \equiv \hbar \equiv G \equiv 1$ we could have the following weird situation. The main thing, can this be confirmed as far as initial GW? 


$$
\begin{aligned}
& V=V_{0} \cdot\left\{\sqrt{\frac{8 \pi G V_{0}}{\gamma(3 \gamma-1)}} \cdot t\right\}^{\sqrt{\frac{\gamma}{4 \pi G}}-\sqrt{\frac{8 \pi G}{\gamma}}} \\
& \underset{l_{\text {Planck }} \equiv \equiv=\xi=1}{\longrightarrow} V_{0} \cdot\left\{\sqrt{\frac{8 \pi V_{0}}{\gamma(3 \gamma-1)}} \cdot t\right\}^{\sqrt{\frac{\gamma}{4 \pi}}-\sqrt{\frac{8 \pi}{\gamma}}} \approx \omega \propto\left(\frac{2}{3 a_{\text {min }}}\right)^{-1 / \pi}
\end{aligned}
$$

The above is with regards to Relic GW generated at the start of inflation.

\section{Summing Up and a Path Forward}

1) I am expecting a strain of Gravitational wave strength of $h \sim 10^{-25}$ to $10^{-26}$;

2) The polarization states to watch, indicating if possible, would be for massive gravitons. The author is trying to ascertain massive graviton formation via the confirmation of the result given by Novello, in [44] about massive gravity, and confirmation of [45] in terms of a weak modification of GR, if feasible. Upon confirmation of that, we will next go to...

3) The ultimate goal would be also to confirm if the multiverse generalization of the Penrose Cyclic Conformal cosmology which the author introduced in [33] is verifiable and does away with the anthropic principle. i.e. we have a statistical averaging procedure for keeping the Planck constant, namely $\hbar$ the same cycle per cycle;

4) As mentioned above, in the beginning of this document also asks about the initial Penrose singularity. For reasons we will state in a future publication, we hold that if the multiverse generalization of the Cyclic Conformal cosmology of Penrose is confirmed, it may aid in falsifying the Penrose singularity. In particular, we hope we can confirm, one way or another [46] [47] so far as to obtain conditions for quantum gravity which may, in part reflect the conditions given in [47] as to primordial GW states and their polarization commensurate with being able to answer if gravity is classical and /or quantum in nature;

5) Finally, we refer to Figure 2 as far as reproduction of structure in the universe as given by [48], and we ask the foundational question of questions as follows;

6) In [48] and [49].

We have wormhole geometries. Is the initial configuration of the Universe in terms of energy conditions, i.e. the negative energy condition at the throat of a worm hole, in any case even partly relevant to the Pre Planckian space-time situation so outlined in this paper?

Finally, as we implied in referencing [33] we find that in order to do away with the Anthropic principle, the following references in terms of Ergodic mixing of the Partition function of the Universe was utilized, as far as a verse. [50] [51] [52]. This is by all accounts a classical physics mixing procedure, as is well understood. Does this application of Ergodic mixing in any way negate the existence of initial conditions pertinent to Quantum gravity? 


\section{Conclusion. Do Virtual Images of Prior Supermassive Black Holes on the CMBR Have a Counterpart in Gravitational Wave Signatures and Signals?}

What we can focus upon in part is the initial phases of when we look at the simpler Starobinsky model as to [53], as to a simpler Cosmology without a Penrose singularity as a start point, as seen in [53], as a compliment to what we have been referencing.

With the Singularity, at the start of Space-time, we have that we will have to revert to Figure 1, of this manuscript, and [2] and likely have only the CMBR signatures as a record of the "ghost" black holes, i.e. no GW values could possibly be obtained from the CMBR, whereas if we have a nonsingular start to expansion of the universe, there are mechanisms which exist which may allow for GW signatures which would exist.

Keep in mind this means that we will likely use the DE construction from [54], whereas the Penrose suggestion we are using can be reviewed using [55]-[61].

We will review next the variant of CCC cosmology used in our model, and conclude with final remarks as to the possibility of obtaining GW signatures of the "phantom" or pre universe black holes.

\section{A Multiverse Generalization of CCC Cosmology}

We are extending Penrose's suggestion of cyclic universes [18], We are extending Penrose's suggestion of cyclic universes, black hole evaporation, and the embedding structure our universe is contained within, This multiverse embeds BHs and may resolve what appears to be an impossible dichotomy. The following is largely taken from [62] and has serious relevance to the final part of the conclusion. That there are no fewer than $N$ universes undergoing Penrose 'infinite expansion' (Penrose) [63] contained in a mega universe structure. Furthermore, each of the $N$ universes has black hole evaporation, with the Hawking

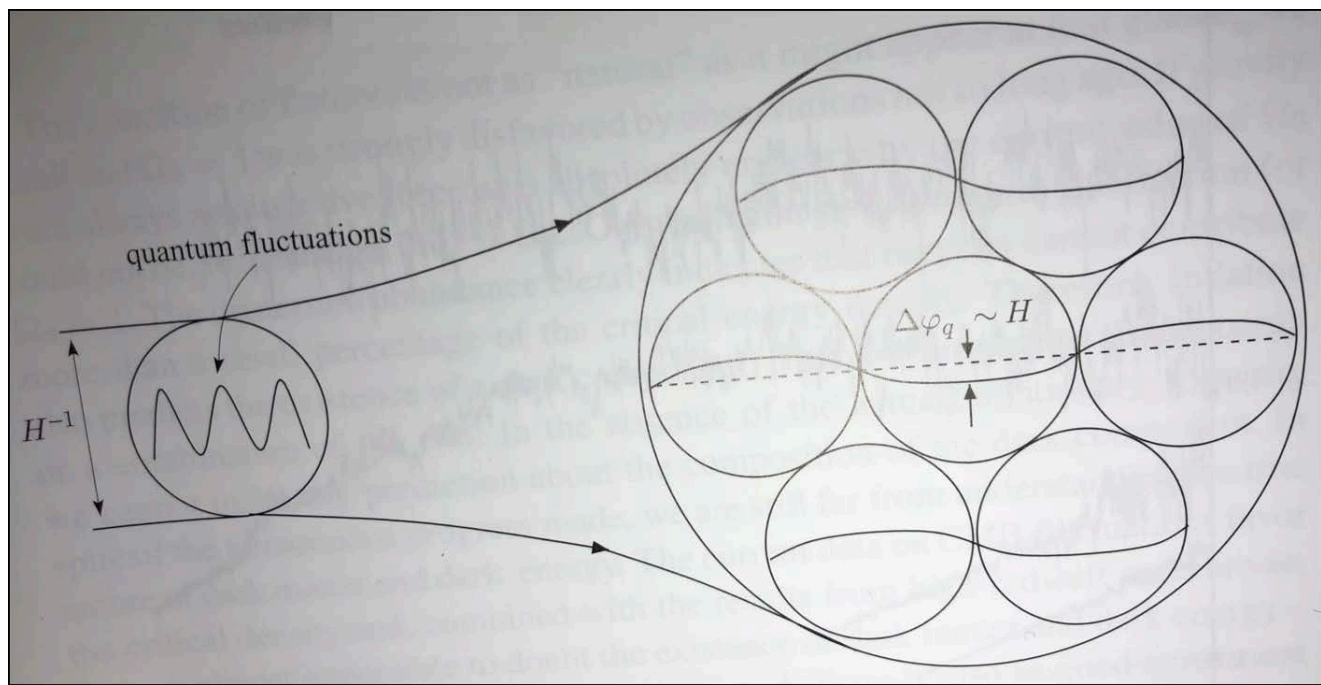

Figure 4. Which is from Mukhanov [48]. 
radiation from decaying black holes. If each of the $N$ universes is defined by a partition function, called $\left\{\Xi_{i}\right\}_{i \equiv N}^{i \equiv 1}$, then there exist an information ensemble of mixed minimum information correlated as about $10^{7}-10^{8}$ bits of information per partition function in the set $\left.\left\{\Xi_{i}\right\}_{i=N}^{i=1}\right|_{\text {before }}$, so minimum information is conserved between a set of partition functions per universe [62].

$$
\left.\left.\left\{\Xi_{i}\right\}_{i \equiv N}^{i \equiv 1}\right|_{\text {before }} \equiv\left\{\Xi_{i}\right\}_{i \equiv N}^{i=1}\right|_{\text {after }}
$$

However, there is non-uniqueness of information put into each partition function $\left\{\Xi_{i}\right\}_{i=N}^{i=1}$. Furthermore Hawking radiation from the black holes is collated via a strange attractor collection in the mega universe structure to form a new big bang for each of the $N$ universes represented by $\left\{\Xi_{i}\right\}_{i=N}^{i=1}$. Verification of this mega structure compression and expansion of information with a non-uniqueness of information placed in each of the $N$ universes favors ergodic mixing treatments of initial values for each of $N$ universes expanding from a singularity beginning. The $n_{f}$ value, will be using $(\mathrm{Ng}, 2008) S_{\text {entropy }} \sim n_{f}$. [64]. How to tie in this energy expression, utilized in the partition function will be to look at the formation of a nontrivial gravitational measure as a new big bang for each of the $N$ universes as by $n\left(E_{i}\right)$ the density of states at a given energy $E_{i}$ for partition function [62].

$$
\left\{\Xi_{i}\right\}_{i=1}^{i=N} \propto\left\{\int_{0}^{\infty} \mathrm{d} E_{i} \cdot n\left(E_{i}\right) \cdot \mathrm{e}^{-E_{i}}\right\}_{i=1}^{i=N}
$$

Each of $E_{i}$ identified with Equation (35) above, are with the iteration for $N$ universes [62] (Penrose, 2006) Then the following holds, by asserting the following claim to the universe, as a mixed state, with black holes playing a major part.

$$
\left.\left.\frac{1}{N} \cdot \sum_{j=1}^{N} \Xi_{j}\right|_{j \text { before nucleation regime }} \stackrel{\text { vacuum nucleation tranfer }}{\longrightarrow} \Xi_{i}\right|_{i \text { fixed after nucleation regime }}
$$

For $N$ number of universes, with each $\left.\Xi_{j}\right|_{j \text { before nucleation regime }}$ for $j=1$ to $N$ being the partition function of each universe just before the blend into the RHS of Equation (36) above for our present universe. Also, each of the independent universes given by $\left.\Xi_{j}\right|_{j \text { before nucleation regime }}$ are constructed by the absorption of one to ten million black holes taking in energy. i.e. (Penrose) [62] [63]. Furthermore, the main point is similar to what was done in [62] in terms of general ergodic mixing.

Claim 2

$$
\left.\left.\Xi_{j}\right|_{j \text { before nucleation regime }} \approx \sum_{k=1}^{\text {Max }} \tilde{\Xi}_{k}\right|_{\text {black holes jth universe }}
$$

What is done in Claim 1 and Claim 2 is to come up as to how a multi dimensional representation of black hole physics enables continual mixing of spacetime [62] [63] largely as a way to avoid the Anthropic principle, as to a preferred set of initial conditions. Claim 2 is to use what is known as CCC cosmology, 
which can be thought of as the following. First. Have a big bang (initial expansion) for the universe. FOR SAY redshift $z=10$, a ten billion years ago, SMBH formation starts. Matter-energy is vacuumed up by the SMBHs, then

$$
\Lambda=c_{1} \cdot[\text { Temp }]^{\beta} \text { if there is quintessence, constant otherwise }
$$

$c_{1}$ is, here a constant. Then the main methodology in the Penrose proposal has been evaluating a change in the metric $g_{a b}$ by a conformal mapping $\hat{\Omega}$ to

$$
\hat{g}_{a b}=\hat{\Omega}^{2} g_{a b}
$$

Penrose's suggestion has been to utilize the following [62] .

$$
\hat{\Omega} \underset{c c c}{\longrightarrow} \Omega^{-1}
$$

In fall into cosmic black hopes has been the main mechanism which the author asserts would be useful for the recycling apparent in Equation (40) above with the caveat that $\hbar$ is kept constant from cycle to cycle as represented by $\hbar_{\text {old cosmology cycle }}=\hbar_{\text {present cosmology cycle }}$ We claim that the invariance of the Planck $\hbar$ combined with Equation (36) above gives a good indication of a uniform mass to a graviton, per cycle, as far as heavy gravity, provided that $\hbar_{\text {old cosmology cycle }}=\hbar_{\text {present cosmology cycle }}$ holds'.

\section{GW Signatures from SM Black Holes from a Prior Universe as a Way to Obtain a Proof of CCC Cosmology}

To do this, would mean we review in dept [4] as mentioned earlier. Do the conditions for [4] show up at the surface of the space-time bubble?

The question delves in the matter of how CMBR patterns reflect GW values. Meaning that a future research project has to be initiated as to do we have conditions at the surface of a nonsingular start to the universe for generating $\mathrm{GW}$ waves?

From [4] we have the following as to generation of GWs, and CMBR.

\section{Quote from page 23}

LENSING-INDUCED B MODES Above we showed that density perturbations do not induce a curl in the polarization. However, that derivation assumed only linear perturbations, in which each Fourier mode of the density field is considered independently. B modes may still arise from the density at higher order. The largest relevant nonlinear effect in the CMB is weak gravitational lensing (or cosmic shear) of the primordial CMB temperature-polarization pattern by density perturbations between us and the CMB surface of last scatter (Zaldarriaga \& Seljak 1998, Lewis \& Challinor 2006). Lensing displaces the temperature and polarization from a given direction $\theta$ at the surface of last scatter to an adjacent position $\theta+\delta \theta$.

End of quote

Do we have additional density perturbations? On the surface of the "bubble" of initial space-time? If the conditions of the quote, as from page 23 of [2] are 
met, we likely have GW signals.

That should be doable as an experimental project on the surface of a bubble, provided higher order perturbative modes are identified.

\section{Funding}

This work is supported in part by National Nature Science Foundation of China grant No. 11375279.

\section{Acknowledgements}

Personal big thank you to Dr. Christian Corda for his outstanding questions which are highlighted in this manuscript.

\section{Conflicts of Interest}

The author declares no conflicts of interest regarding the publication of this paper.

\section{References}

[1] Popławski, N.J. (2020) Black Hole Genesis and Origin of Cosmic Acceleration. https://arxiv.org/abs/1912.02173

[2] An, D., Meissner, K.A., Nurowski, P. and Penrose, R. (2020) Apparent Evidence for Hawking Points in the CMB Sky. arXiv:1808.01740. https://arxiv.org/abs/1808.01740

[3] Rafi, L. (2018) Physicists Think They've Spotted the Ghosts of Black Holes from Another Universe. https://www.livescience.com/63392-black-holes-from-past-universes.html

[4] Kamionkowski, M. and Kovetz, E.D. (2016) The Quest for B Modes from Inflationary Gravitational Waves. Annual Review of Astronomy and Astrophysics, 54, 227-269. https://doi.org/10.1146/annurev-astro-081915-023433 https://www.annualreviews.org/doi/abs/10.1146/annurev-astro-081915-023433

[5] Jeong, D.H. and Kamionkowski, M. (2020) Gravitational Waves, CMB Polarization, and the Hubble Tension. Physical Review Letters, 124, Article ID: 041301 https://doi.org/10.1103/PhysRevLett.124.041301 https://arxiv.org/pdf/1908.06100.pdf

[6] José, M., Senovilla, M. and Garfinkle, David (2015) The 1965 Penrose singularity theorem. https://arxiv.org/abs/1410.5226

[7] Klinkhamer, F.R. (2020) More on the Regularized Big Bang Singularity. Physical Review D, 101, Article ID: 064029. https://doi.org/10.1103/PhysRevD.101.064029 https://arxiv.org/pdf/1907.06547.pdf

[8] Zeldovich, Y.B. (1979) Cosmology and the Early Universe. In: Hawking, S. and Israel, W., Eds., General Relativity, An Einstein Century Survey, Cambridge University Press, New York City, 518-532.

[9] Naselsky, P.D., Novikov, D. and Novikov, I. (2006) The Physics of the Cosmic Microwave Background. Cambridge University Press, Cambridge. https://doi.org/10.1017/CBO9780511536373

[10] Clifford, W. (2018) Theory and Experiment in Gravitational Physics. 2nd Edition, Cambridge University Press, New York City.

[11] Roman, T.A. (1986) Quantum Stress-Energy Tensors and the Weak Energy Condi- 
tion. Physical Review D, 33, 3526-3533. https://doi.org/10.1103/PhysRevD.33.3526

[12] Hawking, S.W. (1994) The Nature of Space and Time. https://arxiv.org/abs/hep-th/9409195

[13] Maciej, D. (2014) Cosmic Jerk and Snap in Penrose's CCC Model. https://arxiv.org/abs/1406.7237

[14] Hindmarsh, M.B., Lüben, M., Lumma, J. and Pauly, M. (2020) Phase Transitions in the Early Universe. https://arxiv.org/abs/2008.09136

[15] Dodelson, S. and Schmitz, F. (2021) Modern Cosmology. 2nd Edition, Academic Press, Cambridge.

[16] Giovannini, M. (1999) Production and Detection of Relic Gravitions in Quintesential Inflationary Models. Physical Review D, 60, Article ID: 123511.

https://doi.org/10.1103/PhysRevD.60.123511 https://journals.aps.org/prd/abstract/10.1103/PhysRevD.60.123511

[17] Low, H.B. and Xiong, C. (2018) Fantasia of a Superfluid Universe, as Part of the Special Volume Put out by World Scientific entitled-In Memory of Kerson Huang. World Scientific, Memorial Volume for Kerson Huang, 55-60. https://doi.org/10.1142/9789813207431_0010

[18] Huang, K., Low, H.B. and Tung, R.S. (2012) Scalar Field Cosmology II: Superfluidity, Quantum Turbulence, and Inflation. International Journal of Modern Physics A, 27, Article ID: 1250154. https://doi.org/10.1142/S0217751X12501540

[19] Huang, K. (2017) A Superfluid Universe. World Scientific, Singapore. https://doi.org/10.1142/10249

[20] Frolov, V. and Zelnikov, A. (2011) Introduction to Black Holes. Oxford University Press, Oxford. https://doi.org/10.1093/acprof:oso/9780199692293.001.0001

[21] Kieffer, C. (2007) Quantum Gravity. 2nd Edition, Oxford Scientific Publications, New York.

[22] Collins, P.D.B., Martin, A.D. and Squires, E.J. (1989) Particle Physics and Cosmology. John Wiley and Sons, New York.

[23] Thanu, P. (2006) An Invitation to Astrophysics. Vol. 8, World Press Scientific, Singapore.

[24] Tolley, A.J. (2015) Cosmological Applications of Massive Gravity. In: Papantonopoulos, E., Ed., Modifications of Einstein's Theory of Gravity at large distances, Vol. 892 Springer, Cham, 203-224.

https://doi.org/10.1007/978-3-319-10070-8_8

[25] D’Amico, G., de Rham, C., Dubosvsky, S., Gabadadze, G., Pirtskhalava, D. and Tolley, A.J. (2011) Massive Cosmologies. Physical Review D, 84, Article ID: 124046. https://arxiv.org/abs/1108.5231 https://doi.org/10.1103/PhysRevD.84.124046

[26] Press, W.H., Teukolsky, S.A., Vetterling, W.T. and Flannery, B.P. (2007) Chapter 4. Integration of Functions. In: Numerical Recipes. The Art of Scientific Computing, 3rd Edition, Cambridge University Press, New York.

[27] Natário, J. (2006) Relativity and Singularities-A Short Introduction for Mathematicians. Resenhas, 6, 309-335.

[28] Barrow, J.D. and Tipler, F.J. (1986) The Anthropic Cosmological Principle. Oxford University Press, Oxford.

[29] Tegmark, M. (1997). On the Dimensionality of Spacetime. Classical and Quantum Gravity, 14, L69-L75. https://doi.org/10.1088/0264-9381/14/4/002 
[30] Freeze, K., Brown, M. and Kinney, W. (2012) The Phantom Bounce, A New Proposal for an Oscillating Cosmology. In: Mercini-Houghton, L, and Vaas, R., Eds., The Arrows of Time, Vol. 172, Springer, Berlin, Heidelberg, 149-156. https://doi.org/10.1007/978-3-642-23259-6_7

[31] Ashtekar, A. (2020) Quantum Gravity in the Sky? Alleviating Tensions in the CMB Using Planck Scale Physics. http://www.icranet.org/images/stories/Meetings/ZM4/presentations/Ashtekar.pdf

[32] Abhay, A., Brajesh, G., Jeong, D.H. and Sreenath, V. (2020) Alleviating the Tension in the Cosmic Microwave Background Using Planck-Scale Physics. Physical Review Letters, 125, Article ID: 051302. https://arxiv.org/abs/2001.11689

[33] Beckwith, A. (2020) How to Obtain a Mass of a Graviton, and Does This Methodology Lead to Voids? Journal of High Energy Physics, Gravitation and Cosmology, 6, 416-439. https://doi.org/10.4236/jhepgc.2020.63032

[34] Klauder, J. (2015) Enhanced Quantization, Particles, Fields \& Gravity. World Press Scientific, Hackensack. https://doi.org/10.1142/9452

[35] Freese, K., Rindler-Daller, T., Spolyar, D. and Valluri, M. (2016) Dark Stars: A Review. Reports on Progress in Physics, 79, Article ID: 066902.

https://doi.org/10.1088/0034-4885/79/6/066902 https://iopscience.iop.org/article/10.1088/0034-4885/79/6/066902

[36] Li, M., Li, X.-D., Wang, S. and Wang, Y. (2015) Dark Energy. Peking University Press-World Scientific, Singapore. https://doi.org/10.1142/9293

[37] Hamber, H.W. and Williams, R.M. (2011) Discrete Wheeler-DeWitt Equation. Physical Review D, 84, Article ID: 104033. https://doi.org/10.1103/PhysRevD.84.104033

[38] Hamber, H.W., Toriumi, R. and Williams, R.M. (2012) Wheeler-DeWitt Equation in 2+1 Dimensions. Physical Review D, 86, Article ID: 084010. https://doi.org/10.1103/PhysRevD.86.084010

[39] Peebles, P.J.E. and Ratra, B. (2003) The Cosmological Constant and Dark Energy. Reviews of Modern Physics, 75, 559-606. https://arxiv.org/abs/astro-ph/0207347 https://doi.org/10.1103/RevModPhys.75.559

[40] Ruchika, C.S. and Sen, A.A. (2004) Model Independent Constraints on Dark Energy Evolution from Low-Redshift Observations. https://arxiv.org/pdf/1806.03943.pdf

[41] Visser, M. (2004) Jerk, Snap, and the Cosmological Equation of State. Classical and Quantum Gravity, 21, 2603-2616. https://arxiv.org/pdf/gr-qc/0309109.pdf https://doi.org/10.1088/0264-9381/21/11/006

[42] Beckwith, A. (2019) Identifying a Kaluza Klein Treatment of a Graviton Permitting a Deceleration Parameter $q(z)$ as an Alternative to Standard DE. Journal of High Energy Physics, Gravitation and Cosmology, 5, 193-217. https://doi.org/10.4236/jhepgc.2019.51011

[43] Cohen-Tannoudji, C., Diu, B. and Laloë, F. (1996) Quantum Mechanics. Wiley, New York. 231-233.

[44] Novello, M. (2005) The Mass of the Graviton and the Cosmological Constant Puzzle. https://arxiv.org/abs/astro-ph/0504505

[45] Corda, C. (2008) Primordial Production of Massive Relic Gravitational Waves from a Weak Modification of General Relativity. Astroparticle Physics, 30, 209-215. https://doi.org/10.1016/j.astropartphys.2008.09.003 https://arxiv.org/abs/0812.0483 
[46] Ahmedov, H. and Aliev, A.N. (2011) More on New Massive Gravity: Exact Solutions. Physical Review Letters, 106, Article ID: 021301.

https://doi.org/10.1103/PhysRevLett.106.021301 https://arxiv.org/abs/1006.4264

[47] Li, F.-Y., Wen, H., Fang, Z.-Y., Li, D. and Zhang, T.-J. (2018) Electromagnetic Counterparts of High-Frequency Gravitational Waves Having Additional Polarization States: Distinguishing and Probing Tensor-Mode, Vector-Mode and Scalar-Mode Gravitons. https://arxiv.org/pdf/1712.00766.pdf

[48] Mukhanov, V. (2005) Physical Foundations of Cosmology. Cambridge University Press, New York. https://doi.org/10.1017/CBO9780511790553

[49] Barros, B.J. and Lobo, F.S.N. (2018) Wormhole Geometries Supported by Three-Form Fields. https://arxiv.org/pdf/1806.10488.pdf

[50] Penrose, R. (2012) Cycles of Time: An Extrardinary New View of the Universe. Vintage, New York.

[51] Poplawski, N. (2011) Cosmological Constant from QCD Vacuum and Torsion. Annalen der Physik, 523, 291-295. https://doi.org/10.1002/andp.201000162 http://arxiv.org/abs/1005.0893v1

[52] Dye, H. (1965) On the Ergodic Mixing Theorem. http://www.ams.org/journals/tran/1965-118-00/S0002-9947-1965-0174705-8/S00029947-1965-0174705-8.pdf

[53] Starobinsky, A.A. (1980) A New Type of Isotropic Cosmological Models without Singularity. Physics Letters B, 91, 99-102. https://doi.org/10.1016/0370-2693(80)90670-X

[54] Overduin, J. and Wesson, P.S. (2008) The Light Dark Universe, Light from Galaxies, Dark Matter and Dark Energy. World Scientific, Singapore. https://doi.org/10.1142/7001

[55] Hajian, A. (2010) Are There Echoes From The Pre-Big Bang Universe? A Search for Low Variance Circles in the CMB Sky. The Astrophysical Journal, 740, 52. https://doi.org/10.1088/0004-637X/740/2/52

[56] DeAbreu, A., Contreras, D. and Scott, D. (2015) Searching for Concentric Low Variance Circles in the Cosmic Microwave Background. Journal of Cosmology and Astroparticle Physics, 2015, 031. https://doi.org/10.1088/1475-7516/2015/12/031

[57] Gurzadyan, V.G. and Penrose, R. (2010) More on the Low Variance Circles in CMB Sky. arXiv:1012.1486.

[58] Gurzadyan, V.G. and Penrose, R. (2016) CCC and the Fermi Paradox. The European Physical Journal Plus, 131, Article No. 11. https://doi.org/10.1140/epjp/i2016-16011-1

[59] Gurzadyan, V.G. and Penrose, R. (2013) On CCC-Predicted Concentric Low-Variance Circles in the CMB Sky. The European Physical Journal Plus, 128, Article No. 22. https://doi.org/10.1140/epjp/i2013-13022-4

[60] Jow, D.L. and Scott, D. (2020) Re-Evaluating Evidence for Hawking Points in the CMB. Journal of Cosmology and Astroparticle Physics, 2020, 021. https://doi.org/10.1088/1475-7516/2020/03/021

[61] Roger, P. (2006) Hawking Points in the Cosmic Microwave Background-A Challenge to the Concept of Inflation.

[62] Beckwith, A. (2014) Analyzing Black Hole Super-Radiance Emission of Particles/Energy from a Black Hole as a Gedankenexperiment to Get Bounds on the Mass of a Graviton. Advances in High Energy Physics, 2014, Article ID: 230713. 
https://doi.org/10.1155/2014/230713

[63] Penrose, R. (2011) Cycles of Time-An Extraordinary New View of the Universe. Vintage, New York.

[64] Ng, Y. (2008) Spacetime Foam: From Entropy and Holography to Infinite Statistics and Nonlocality. Entropy, 10, 441-461. https://doi.org/10.3390/e10040441 